\title{
The role of virus-derived small interfering RNAs in RNA silencing in plants
}

\author{
ZHU Hui \& GUO HuiShan* \\ State Key Laboratory of Plant Genomics and National Center for Plant Gene Research, Institute of Microbiology, \\ Chinese Academy of Sciences, Beijing 100101, China
}

Received November 30, 2011; accepted December 28, 2011

\begin{abstract}
Antiviral defense is one of the important roles of RNA silencing in plants. Virus-derived small interfering RNAs (vsiRNAs) are found in the infected host cells, indicating that the host RNA silencing machinery can target viral RNAs for destruction. With the development of high-throughput sequencing of vsiRNAs, recent genetic studies have shed light on the origin and composition of vsiRNAs and their potential functions in the regulation of gene expression. Here, we briefly describe the origin and biogenesis of vsiRNAs, and review the recent discoveries regarding vsiRNA-mediated RNA silencing of viral genomes and host transcripts. This will better our understanding of virus pathogenicity and RNA silencing-related host-pathogen interactions in plants.
\end{abstract}

vsiRNA, RNA silencing, virus pathogenicity, gene regulation

Citation: Zhu H, Guo H S. The role of virus-derived small interfering RNAs in RNA silencing in plants. Sci China Life Sci, 2012, 55: 119-125, doi: $10.1007 / \mathrm{s} 11427-012-4281-3$

RNA silencing is a highly conserved mechanism that operates in most eukaryotes. One of the major features of RNA silencing is the production of small RNAs of 21-30 nucleotides (nt) in length which can regulate gene expression in a sequence-specific manner. The small RNAs are grouped into three classes: small interfering RNAs (siRNAs), microRNAs (miRNAs) and Piwi-associated interfering RNAs (piRNAs). Generally, RNA silencing can be triggered by double-stranded RNA (dsRNA) or a hairpin-like fold-back structure formed through intramolecular basepairing. In the silencing initiation stage, a double-stranded RNA precursor can be recognized and processed into small RNAs by Dicer or Dicer-like (DCL) proteins [1-3]. Then, the maturely-processed small RNAs are recruited by the Argonaute (AGO) protein to form an RNA-induced multi-subunit silencing complex (RISC). Finally, these small RNAs guide RISC to regulate targets in a sequence-specific manner.

*Corresponding author (email: guohs@im.ac.cn)
This causes transcriptional silencing through induction of methylation, or post-transcriptional gene silencing (PTGS) through sequence-specific cleavage of target RNA and translational inhibition [1-3]. RNA silencing pathways in plants play important roles in defense against invading viruses and foreign transposons, in the regulation of chromatin modification and in the control of gene expression to guarantee the normal development of plants.

Two lines of evidence support the role of RNA silencing as an antiviral defense mechanism in plants. On the one hand, a large number of virus-derived small RNAs are found in the infected host plants, indicating that the host RNA silencing mechanism can target viral RNA [4]. This is called virus infection-induced gene silencing (VIGS). On the other hand, many plant viruses encode silencing suppressors to counter host antiviral defense mechanisms based on RNA silencing [5-7]. This provides additional evidence for the antiviral nature of RNA silencing. The production of a large number of virus-derived small interfering RNAs 
(vsiRNAs) is the most remarkable feature of virus-induced gene silencing [4,8-13]. Current research indicates that vsiRNAs are derived either from the dsRNAs formed during the virus replication stage or from single-stranded viral RNAs (ssRNAs) with a hairpin-like fold-back structure [8,10,14-16]. Both of these forms of viral RNA can be processed into vsiRNAs of a specific size by distinct DCLs proteins. However, the cleavage of viral RNAs by DCLs is not in itself sufficient to suppress virus replication. Other key components of silencing, such as Ago and RNA-dependent RNA polymerase (RDR), are also required for effective virus silencing $[17,18]$. It has been demonstrated that VIGS-mediated viral RNA inactivation is likely based on the vsiRNA-mediated cleavage of viral RNA $[8,12]$ or of viral satellite RNA [19]. However, it cannot be excluded that the antiviral silencing mechanism also acts at the level of translational regulation.

With respect to vsiRNA-mediated RNA silencing, several regulation models exist according to current research results, one of which is that vsiRNAs can guide the cleavage of genomic viral RNA. Moreover, virus infection can cause distinct phenotypic symptoms, which are often related to changes in the level of expression of host genes [20-23]. In view of the complexity of vsiRNA sequences, it is reasonable to consider that vsiRNAs can target and regulate host transcripts post-transcriptionally. In this review we mainly summarize the recent research advances with a focus on vsiRNA-mediated RNA silencing, including that of viral genomic RNA and host transcript RNA.

\section{Biogenesis of virus-derived small RNAs in plants}

\subsection{Origin of viral siRNAs}

Plant virus genomes serve as both the trigger and target of post-transcriptional gene silencing (PTGS). In early models, it was widely thought that replication of positive-strand RNA viruses occurs via double-stranded intermediates which were likely the trigger activating the production of vsiRNAs [24]. This meant that there should be the same amount of vsiRNAs derived from positive- and negative-sense strands in the infected host plant cells. However, by molecular cloning of small RNAs derived from several positive-strand plant RNA viruses, Molnar et al. [13] found that the production of the vsiRNAs had a strong bias towards the genomic-sense strand. This challenged the viewpoint of genome-length viral dsRNA being the unique precursor. Molnar et al. [13] also proposed that highly structured, single-stranded viral RNAs could be processed into vsiRNAs to trigger RNA silencing. It is well-known that, as far as positive-strand viruses are concerned, virus replication is associated with extensively rearranged intracellular membranes, and replication intermediates are assembled with many viral RNA-dependent RNA polymerase (RdRp) molecules [25]. Thus, the possibility that dsRNA replication intermediates are processed into vsiRNAs is reduced, therefore the great number of vsiRNAs found in virusinfected tissues could not only be derived from dsRNA replication intermediates. Although each position on the viral genome is a potential cleavage site in producing vsiRNA, there exists a distinction between "hot" and "non-hot" regions. It has been suggested that dsRNA-like secondary structures within the single-stranded viral RNA are more likely to be the main source of vsiRNAs than dsRNA replication intermediates [8,10,14-16]. Plant DNA viruses, which have a dsDNA intermediate stage, produce an abundance of vsiRNAs from RNA transcription units [26]. Most of the vsiRNAs related to geminiviruses (plant DNA viruses which have single-stranded DNA genomes) are probably derived from fold-back structures within RNA transcription units $[27,28]$.

\subsection{Biogenesis of vsiRNAs}

With the discovery of endogenous small RNAs in diverse eukaryotes, scientists have done extensive genetic and biochemical research into their biogenesis in many model systems, concentrating especially on the variety of effector proteins participating in the small RNA-mediated silencing pathway. The current knowledge on vsiRNA biogenesis in plants comes mainly from genetic analysis in the model plant Arabidopsis.

Viral dsRNA, or highly-structured regions in ssRNA, can be processed into specifically-sized vsiRNAs by distinct DCLs. DCL2 and DCL4 play key roles in the generation of vsiRNAs derived from positive-strand RNA viruses, as determined using loss-of-function mutants in Arabidopsis DCLs. Plants infected with positive-strand RNA viruses mainly accumulate 21-nt vsiRNAs processed by DCL4 $[15,29,30]$. However, there appears to be a size shift of the vsiRNAs to DCL2-dependent 22-nt species in the absence of DCL4. For example, host plants infected with Turnip crinkle virus (TCV) only accumulate DCL2-dependent 22-nt vsiRNA. DCL4-dependent 21-nt vsiRNAs were detected only following infection with TCV $\triangle \mathrm{P} 38$ (which lacks the viral suppressor P38), suggesting that P38 encoded by wild-type TCV interferes with DCL4 during normal infections and hence DCL2 takes over the antiviral defense [31]. DCL3 produces 24-nt vsiRNAs only in $d c l 2 / d c l 4$ double mutants, thus seeming to play a minor role in this process $[15,29-31]$. It is currently believed that DCL1 is only indirectly involved in vsiRNAs generation, since all the DCLs are in a self-balanced system. In the case of plant viruses with DNA genomes, all four DCLs participate in the generation of vsiRNAs, thus differing from RNA viruses, DCL3 being relatively the most active antiviral dicer and DCL1 seeming to assist the other DCLs [26,32].

The vsiRNAs generated from DCL proteins acting di- 
rectly on the invading viral genome are called primary vsiRNAs. vsiRNAs can guide AGO to cleave the viral genome, and the resulting cleaved viral fragments are possibly amplified into dsRNAs by plant RDRs. The newly-synthesized dsRNAs are recognized and processed by DCL into new vsiRNAs called secondary visRNAs, which is similar to the generation of RDR6-dependent tasiRNAs (trans-acting siRNAs) [33-35]. Arabidopsis possesses six RDR proteins. It has been shown that RDR1 and RDR6 might take part in the biogenesis of secondary vsiRNAs [36]. Small RNA high-throughput sequencing technology has promoted the study of the roles of host RDRs in the biogenesis of vsiRNAs $[11,14,15]$. Recently, Wang et al. [14] pointed out that RDR1 and RDR6 exhibited specificity in targeting the genome sequences of Cucumber mosaic virus (CMV) in amplifying secondary vsiRNAs. RDR1 preferentially amplified the 5'-terminal siRNAs of the three genomic RNAs of CMV. However, an increased production of vsiRNAs targeting the $3^{\prime}$ half of RNA3 detected in $r d r 1$ mutant seemed to be RDR6-dependent.

It is necessary for vsiRNAs to bind and guide AGOs to cleave target genes in order to further amplify secondary vsiRNA precursors. Arabidopsis encodes 10 AGO proteins. A study on Arabidopsis ago mutants found that certain AGO proteins participate in vsiRNAs biogenesis. AGO1 immunoprecipitates from Arabidopsis infected by CMV, TCV and Turnip yellow mosaic virus (TYMV) contained vsiRNAs [17,18]. Moreover, CMV-derived vsiRNAs have been detected in AGO2 and AGO5 immunoprecipitates [37,38]. Additionally, in Arabidopsis ago7 or ago2 mutants, the accumulation level of TCV viral RNA is higher than that in wild-type plants [37,39]. All the studies above indicate that vsiRNAs likely guide the AGO-containing RISCs to specifically cleave viral RNAs and promote downstream biogenesis of RDR-dependent secondary vsiRNAs, which finally trigger the efficient silencing. This viewpoint has been confirmed in recent research using plants infected with 2b-deficient CMV (CMV- $\Delta 2$ b), in which AGO1 and AGO2 were shown to act downstream in the biogenesis of secondary vsiRNAs in a non-redundant and cooperative manner [40].

\section{Molecular basis of the vsiRNA-mediated an- tiviral effect}

The restriction of virus replication is not effected simply through the production of primary vsiRNAs by DCL-dependent action on the infecting viral genome. Both genetic and biochemical data show that the formation of vsiRNA-containing functional RISCs is significant for effective antiviral defense [40]. Currently, it is unclear if all the vsiRNAs produced in the host cell can be incorporated into AGO-containing RISCs, and it remains to be established whether vsiRNAs can be recruited into all the AGO family members. By analyzing Tobacco mosaic virus (TMV)-Cg- derived 21-nt siRNA populations in infected Arabidopsis, Qi et al. [16] found there existed four classes of vsiRNAs with distinct 5'-terminal nucleotides. The vsiRNAs with A or $\mathrm{U}$ as their initiation base exceeded in number those with $\mathrm{G}$ or C. It has been verified that distinct AGOs in Arabidopsis show a bias towards the $5^{\prime}$ terminal nucleotides when recruiting endogenous siRNAs $[38,41,42]$. However, it remains to be clarified whether AGOs obey the same rules when recruiting vsiRNAs in the antiviral process.

The current experimental evidence on vsiRNA-mediated antiviral functions indicates that the VIGS-mediated antiviral role is primarily based on the sequence-specific cleavage of viral RNA guided by vsiRNAs [12]. Using recombinant Tobacco rattle virus (TRV), researchers found that TRV infection of plants was associated with the accumulation of vsiRNAs. These targeted recombinant RNA for degradation, as manifested by a decreased virus titer over time [11]. Viral genomes possess hot spots for generating vsiRNAs, as shown by high-throughput deep sequencing techniques [8]. To understand the biological role of these hot spots, researchers carried out analyses of vsiRNAs and their downregulatory effect on their corresponding hot spot and non-hot spot regions. Their results show that vsiRNAs generated from hotspots, despite their much greater abundance, do not show a greater efficiency than those from non-hotspots regions [8]. It has been shown that most of the Cymbidium ringspot virus (CymRSV) vsiRNAs (including those derived from hot spot regions) are primary vsiRNAs. This study, on the one hand, identified that vsiRNAs derived from the direct cleavage of viral genome by DCLs were not efficient in the down-regulation of accumulation of viral RNAs; on the other hand, it suggested that the hot spot regions producing vsiRNAs were not necessarily the effective regions for the vsiRNA-AGO complex to target. Furthermore, vsiRNAs entering into AGO complexes were not necessarily playing a role in the inactivation of viruses [12]. The high level of secondary structure in certain viruses, virus satellite RNAs and viroids possibly obstruct the targeting effect of the vsiRNAs-AGO complex. Therefore, the accessibility of target sites may also determine the efficiency of antiviral RNA silencing [43].

Many viruses encode silencing suppressor proteins. Different suppressors interfere with or suppress different components or steps of the silencing pathway. Using suppressor-deficient mutant virus to infect plants has become an effective experimental means of identifying the components of antiviral RNA silencing [31,44-46]. For example, Wang et al. $[14,40]$ established the roles of Arabidopsis RDRs in silencing-mediated virus resistance by using CMV- $\Delta 2$ b (deficient in the expression of suppressor $2 b$ ). The resistance to CMV- $\Delta 2 \mathrm{~b}$ in Arabidopsis was mainly associated with the amplification of vsiRNAs generated by a RDR6-dependent pathway. Production of the secondary vsiRNAs targeting CMV- $\Delta 2 \mathrm{~b}$ required Suppressor of gene silencing 3 (SGS3) and DCL4 in addition to RDR6 [40]. In addition, examina- 
tion of a series of AGO-related mutants, combined with co-immunoprecipitation and deep sequencing, verified the essential requirement for $\mathrm{AGO} 1$ and $\mathrm{AGO} 2$ in the defense against $C M V-\Delta 2$ b. It also verified that $21-n t$, but not $22-n t$, secondary vsiRNAs utilized the two cooperative AGOs to mediate the antiviral RNA silencing in Arabidopsis [40].

A comparison of the ratio of accumulated viral RNA to that of vsiRNA may preliminarily reveal the antiviral role of vsiRNAs. However, to date it has not been reported that a specific virus-derived small RNA can target a viral genome to induce resistance. We previously cloned the siRNAs derived from the satellite RNA (satsiRNA) of SD-CMV (a severe field Shan-Dong strain) [47], and have recently examined the biological function of satsiRNAs. Our results indicate that these satsiRNAs can combine with AGO proteins in host plants. The most frequently cloned satsiRNA, named satsiR-12 that targets the 3' UTR of CMV RNA, triggers the RDR6-dependent antiviral silencing pathway [48]. Firstly, using a plant transient expression system, we found the expression of artificial satsiR-12 (asatsiR-12) could mediate the cleavage of a GFP-sensor harboring the satsiR-12 target site. Moreover, using CMV- $\Delta 2$ b-infected transgenic plants expressing asatsiR-12, we found that asatsiR-12 was able to reduce the accumulation of CMV RNAs. Also, we verified that asatsiR-12-mediated reduction of viral RNAs in the presence of RDR6 was inhibited by the $2 \mathrm{~b}$ suppressor [48]. A high concentration of replicating satRNAs could reduce the accumulation of viral RNAs in $N$. benthamiana on later infection by $C M V-\Delta 2 b$ [49]. By introducing mutations into the region generating satsiR-12, we confirmed that satsiR-12 was indeed involved in the satRNA-mediated reduction in CMV RNAs. Our findings provided the first demonstration that viral satellite RNA-derived siRNAs could mediate silencing against their helper virus [48].

\section{Potential action of vsiRNAs in regulation of host transcript expression}

Early studies suggested that virus disease symptoms were partly attributed to the activity of virus-encoded silencing suppressors. These disturbed certain important endogenous siRNAs that regulated the developmental process of the host, since the binding of siRNAs is the most common mechanism used by most of the suppressors of virus silencing [50-52]. Recent studies have led us to believe that some of the vsiRNAs may target host transcripts for posttranscriptional regulation. Although many host transcripts that could potentially be targeted by vsiRNAs have been found using bioinformatics, only a few studies have provided experimental evidence to verify the targeting of host genes during vsiRNAs-mediated RNA silencing.

A blast search with the full-length sequence of the RG1 strain of Potato spindle tuber viroid (PSTVd-RG1) found that large numbers of sequences from several plant species shared 19-20 nt identities with the PSTVd sequence. Most of these plant sequences corresponded to the virulence-regulating region of PSTVd. Among the identified plant sequences, there were at least two encoding putative transcription factors and one encoding a putative chromo-domain helicase DNA-binding protein [53]. This result suggested the possibility that siRNAs derived from the virulence-regulating region of PSTVd may cause silencing of host regulatory genes.

Researchers compared the 35S RNA leader sequence of Cauliflower mosaic virus (CaMV, a double-stranded DNA virus) with Arabidopsis cDNAs and ESTs to look for possible 18-25 nt microhomologies [26]. Allowing a maximum of two mismatches, three Arabidopsis genes, including At $\lg 76950$, were found. Sequence alignment revealed a near-perfect complementarity between the 5' UTR of At $\lg 76950$ and a 20-nt sequence located in the 35S RNA leader sequence. This implied that these vsiRNAs (sRCC1) derived from the 35S RNA leader sequence could serve as bona fide siRNAs to mediate the cleavage of the At $1 g 76950$ transcript during infection. Semiquantitative RT-PCR (sQPCR) analysis indicated that accumulation of At $1 g 76950$ mRNA was greatly reduced in Arabidopsis infected with CaMV. A transient expression experiment using Agrobacterium infiltration into $\mathrm{CaMV}$-infected plants revealed that CaMV infection facilitated sequence-specific down-regulation of the infiltrated reporter gene sequence harboring the sRCC1 target site. These findings provided strong evidence for the reduction of Atlg76950 levels in Arabidopsis being due to the direct action of CaMV-derived sRCC1.

Using a similar procedure for miRNA target prediction, 4784 host genes were predicted to be the potential targets of the siRNAs of TMV-Cg (a crucifer-infecting strain of TMV) [16]. However, vsiRNAs-mediated cleavage sites were detected only in a few predicted target genes by 5'RACE. Many factors might affect the functionality of vsiRNAs and hence restrict their regulatory potential on host targets in vivo. Moreover, various virus-encoded silencing suppressors could also suppress the activity of vsiRNAs on the regulation of putative host targets. In addition, the extent to which vsiRNAs can regulate host gene expression might also depend on the abundance of vsiRNAs.

Recently, two research groups simultaneously confirmed, for the first time, that siRNAs derived from viral satellite RNA could directly regulate the expression of a host gene and modulate the virus disease symptoms. CMV-Y satellite RNA (Y-Sat) can induce yellowing symptoms in infected tobacco plants, of which the molecular mechanism is not understood. Previous studies have shown that the nucleotide sequence responsible for the yellowing was located in a 24-nt region of the Y-Sat genome, called the "yellow domain" [54-57]. The group led by Wang first looked for the target genes containing complementary regions to the "yellow domain" with a blast search [58]. They found that a 
22-nt complementary region was located in the sequence of the gene involved in chlorophyll biosynthesis, the CHLI gene, which encodes a magnesium chelatase subunit. Small RNA sequencing and 5'RACE analyses confirmed that Y-sat-derived siRNAs could target the 22-nt complementary sequence for the cleavage of CHLI mRNA. Transformation of tobacco with an RNAi construct targeting the CHLI gene was able to induce Y-Sat-like symptoms. Moreover, the symptoms induced by Y-Sat infection could be completely prevented by expressing a mutated CHLI gene resistant to "yellow domain"-mediated cleavage in tobacco [58]. All these results indicate that Y-Sat-induced symptoms are caused by the vsiRNAs-mediated silencing of CHLI. Similarly, Shimura et al. [62] also revealed the molecular mechanism of the yellowing symptom induced by Y-Sat. They found that transgenic $N$. benthamiana plants that expressed the inverted-repeat sequence of Y-Sat also developed yellowing symptom similar to the Y-Sat-infected plants. They also showed that the expression level of CHLI was down-regulated in the above transgenic plants and in Y-Satinfected plants. Finally, they verified that Y-Sat-derived vsiRNAs could specifically target the 22-nt sequence in CHLI mRNA and therefore down-regulate CHLI mRNA, thus inducing the yellowing symptom by impairing the chlorophyll biosynthesis pathway.

\section{Role of vsiRNAs in balancing the interaction between host, helper virus and satellite RNA}

The effects of satRNAs on the pathogenicity of their helper virus depend on the strain of helper virus, satRNA and host plant. Increasingly, evidence in recent years has shown that RNA silencing mechanism of host plants plays important roles in the pathogenicity and evolution of satRNAs. The interaction among the three (satRNA, helper virus, host) during RNA silencing is rather complex. However, the interaction is always in a state of relative balance, so that any one of the three would not be eliminated and could therefore continue to contribute to the long co-evolutionary process. Many cases referred to above show clearly that host plants, once invaded by virus, will activate their own RNA silencing system to target the foreign nucleic acid $[8,11,12]$, and the virus will correspondingly express an RNA silencing suppressor to restrict the host defense system [59-61]. Also, viral satRNA can utilize the host RNA silencing system to regulate the expression of certain host genes and thus induce symptoms [58,62]. Pantaleo and Burgyan [19] also pointed out that CymRSV could use an RNA silencing mechanism to control the accumulation level of satRNA, while satRNA might assist helper virus to resist the silencing-based defense system of host plants [63,64].

Our latest results found that CMV-satRNA (SD strain) could reduce the expression level of the suppressor $2 \mathrm{~b}$ protein and thus attenuate the symptoms caused by CMV [49].
This perfectly illustrated the relationships between host plant, helper virus and satRNA in RNA silencing. SD-satRNA reduced the accumulation of the $2 b$ coding sequence, which meant that the host silencing that was suppressed by $2 \mathrm{~b}$ partially recovered. Therefore, the plants infected with wild-type CMV did not display the symptom phenotype relevant to $2 b$ in the presence of SD-satRNA [49]. In addition, lower levels of CMV-specific vsiRNAs were detected in plants infected with SD-satRNA in both the presence or absence of $2 \mathrm{~b}$ protein. In contrast, abundant SD-satRNA-derived vsiRNAs were detected [49]. The result indicated that SD-satRNA likely interfered with the silencing of helper virus by recruiting host RNA silencing components, which actually prevented the viral genome from being targeted by the RNA silencing pathway. Our results suggested that the host silencing mechanism was involved in the pathogenicity of satRNA. In other words, the pathogenicity of SD-satRNA is a result of a complex interaction among SD-satRNA, helper virus and host. Our recent research results further indicated that satRNAderived vsiRNAs were involved in the interaction between the three (satRNA, helper virus, host). On the one hand, the host defense mechanism-based RNA silencing triggered by CMV infection could target satRNA for the production of satsiRNAs. Among these satsiRNAs, satsiR-12 mediated the host RDR6-dependent antiviral silencing by targeting the 3' UTR of CMV RNA. Moreover, the satsiR-12-mediated RDR6-dependent antiviral defense response was inhibited by the suppressor $2 b$ [48]. On the other hand, the abundant replication of satRNA affected the accumulation of $2 b$ coding RNA [49]. Reducing the activity of the $2 b$ silencing suppressor could attenuate the damage to plant development that is regulated by endogenous small RNAs, which not only ensures the replication of virus in plants but also guarantees that the host is not destroyed. The siRNA-mediated silencing and suppressor-mediated anti-silencing reflect the "defense-suppression-counter suppression" cycle between plant hosts and pathogens, illustrating the endless competition for survival.

\section{Conclusion}

Regarding the roles of vsiRNAs in antiviral defense, this review mainly discusses the process of PTGS caused by vsiRNAs that guide RISC to target a viral genome or a subviral RNA. Recent research has indicated that transcriptional gene silencing (TGS) caused by small RNA-guided methylation is also a defense mode against DNA viruses [65]. Tomato yellow leaf curl China virus (TYLCCNV), a member of the geminiviruses, has a satellite RNA called DNA $\beta$ (betasatellite). The latest study from Zhou's lab suggests that the pathogenicity factor $\beta \beta C 1$ encoded by $\beta$ satellite stabilized geminivirus/ $\beta$ satellite-complexes through the inhibition of methylation-mediated TGS and thereby in- 
duced disease symptoms [66]. At present, evidence for the role of vsiRNAs in translational repression is limited. Blevins et al. [67] found that vsiRNAs derived from the leader sequence of CaMV did not restrict virus replication, but served as a decoy diverting the silencing machinery from the viral promoter and coding regions. More studies are needed to find out whether other plant viruses utilize RNA decoys to evade RNA silencing mechanisms. The biggest effect of RNA silencing on antiviral defense seems to be the restriction of systemic spread of the virus. Certain key issues remain unresolved, such as the nature of the systemic silencing signal molecules, and how they are generated. Future investigation to address these issues will be of great interest.

Do vsiRNAs specifically regulate the expression of host genes? Many efforts have been made to answer this question. Initially, bioinformatics predicted large numbers of potential host transcripts of vsiRNAs. Some of the potential targets were preliminarily identified utilizing sensor constructs related to foreign reporter genes and 5'RACE. Nowadays, high-throughput deep sequencing technology has made it possible to search for the authentic vsiRNAs targets in the context of the whole genome. As described in this review, although significant progress in the understanding of vsiRNA metabolism and function has been made, our understanding of the vsiRNA-guided silencing pathway is far from complete, and many important issues need to be addressed.

In the course of exploring vsiRNA-mediated virus resistance, research evidence has increasingly revealed that the RNA silencing mechanisms of host plants play important roles in the pathogenicity of satRNA and in their co-evolution with the helper virus. vsiRNAs play a significant role in the homeostatic interactions among the host, virus and satRNA in determining the final outcome of a virus infection. Further study on viruses and their satRNAs, as well as on vsiRNAs, will lead to a better understanding of how hosts and viruses interact with each other to achieve co-evolutionary survival.

1 Chen X. Small RNAs-secrets and surprises of the genome. Plant J, 2010, 61: 941-958

2 Ghildiyal M, Zamore P D. Small silencing RNAs: an expanding universe. Nat Rev Genet, 2009, 10: 94-108

3 Chapman E J, Carrington J C. Specialization and evolution of endogenous small RNA pathways. Nat Rev Genet, 2007, 8: 884-896

4 Hamilton A J, Baulcombe D C. A species of small antisense RNA in posttranscriptional gene silencing in plants. Science, 1999, 286: 950-952

5 Burgyan J. Role of silencing suppressor proteins. Methods Mol Biol, 2008, 451: 69-79

6 Ding S W, Voinnet O. Antiviral immunity directed by small RNAs. Cell, 2007, 130: 413-426

7 Voinnet O, Pinto Y M, Baulcombe D C. Suppression of gene silencing: a general strategy used by diverse DNA and RNA viruses of plants. Proc Natl Acad Sci USA, 1999, 96: 14147-14152

8 Szittya G, Moxon S, Pantaleo V, et al. Structural and functional analysis of viral siRNAs. PLoS Pathog, 2010, 6: e1000838
9 Qu F. Antiviral role of plant-encoded RNA-dependent RNA polymerases revisited with deep sequencing of small interfering RNAs of virus origin. Mol Plant Microbe Interact, 2010, 23: 1248-1252

10 Donaire L, Wang Y, Gonzalez-Ibeas D, et al. Deep-sequencing of plant viral small RNAs reveals effective and widespread targeting of viral genomes. Virology, 2009, 392: 203-214

11 Donaire L, Barajas D, Martinez-Garcia B, et al. Structural and genetic requirements for the biogenesis of tobacco rattle virus-derived small interfering RNAs. J Virol, 2008, 82: 5167-5177

12 Pantaleo V, Szittya G, Burgyan J. Molecular Bases of Viral RNA Targeting by Viral Small Interfering RNA-Programmed RISC. J Virol, 2007, 81: 3797-3806

13 Molnar A, Csorba T, Lakatos L, et al. Plant Virus-derived small interfering RNAs originate predominantly from highly structured single-stranded viral RNAs. J Virol, 2005, 79: 7812-7818

14 Wang $\mathrm{X} \mathrm{B}, \mathrm{Wu} \mathrm{Q}$, Ito $\mathrm{T}$, et al. RNAi-mediated viral immunity requires amplification of virus-derived siRNAs in Arabidopsis thaliana. Proc Natl Acad Sci USA, 2010, 107: 484-489

15 Garcia-Ruiz H, Takeda A, Chapman E J, et al. Arabidopsis RNA-dependent RNA polymerases and Dicer-like proteins in antiviral defense and small interfering RNA biogenesis during Turnip Mosaic Virus infection. Plant Cell, 2010, 22: 481-496

16 Qi X, Bao F S, Xie Z. Small RNA deep sequencing reveals role for Arabidopsis thaliana RNA-dependent RNA polymerases in viral siRNA biogenesis. PLoS ONE, 2009, 4: e4971

17 Azevedo J, Garcia D, Pontier D, et al. Argonaute quenching and global changes in Dicer homeostasis caused by a pathogen-encoded GW repeat protein. Genes Dev, 2010, 24: 904-915

18 Zhang X, Yuan Y R, Pei Y, et al. Cucumber mosaic virus-encoded 2b suppressor inhibits Arabidopsis Argonaute1 cleavage activity to counter plant defense. Genes Dev, 2006, 20: 3255-3268

19 Pantaleo V, Burgyan J. Cymbidium ringspot virus harnesses RNA silencing to control the accumulation of virus parasite satellite RNA. J Virol, 2008, 82: 11851-11858

20 Golem S, Culver J N. Tobacco mosaic virus induced alterations in the gene expression profile of Arabidopsis thaliana. Mol Plant Microbe Interact, 2003, 16: 681-688

21 Whitham S A, Yang C, Goodin M M. Global impact: elucidating plant responses to viral infection. Mol Plant Microbe Interact, 2006, 19: $1207-1215$

22 Agudelo-Romero $\mathrm{P}$, Carbonell $\mathrm{P}$, de la Iglesia $\mathrm{F}$, et al. Changes in the gene expression profile of Arabidopsis thaliana after infection with Tobacco etch virus. Virol J, 2008, 5: 92

23 Havelda Z, Varallyay E, Valoczi A, et al. Plant virus infection-induced persistent host gene downregulation in systemically infected leaves. Plant J, 2008, 55: 278-288

24 Ahlquist P. RNA-dependent RNA polymerases, viruses, and RNA silencing. Science, 2002, 296: 1270-1273

25 den Boon J A, Ahlquist P. Organelle-like membrane compartmentalization of positive-strand RNA virus replication factories. Annu Rev Microbiol, 2010, 64: 241-256

26 Moissiard G, Voinnet O. RNA silencing of host transcripts by cauliflower mosaic virus requires coordinated action of the four Arabidopsis Dicer-like proteins. Proc Natl Acad Sci USA, 2006, 103: 19593-19598

27 Vanitharani R, Chellappan P, Fauquet C M. Geminiviruses and RNA silencing. Trends Plant Sci, 2005, 10: 144-151

28 Chellappan P, Vanitharani R, Fauquet C M. Short Interfering RNA accumulation correlates with host recovery in DNA virus-Infected hosts, and gene silencing targets specific viral sequences. J Virol, 2004, 78: 7465-7477

29 Fusaro A F, Matthew L, Smith N A, et al. RNA interference-inducing hairpin RNAs in plants act through the viral defence pathway. EMBO Rep, 2006, 7: 1168-1175

30 Bouche N, Lauressergues D, Gasciolli V, et al. An antagonistic function for Arabidopsis DCL2 in development and a new function for DCL4 in generating viral siRNAs. EMBO J, 2006, 25: 3347-3356

31 Deleris A, Gallego-Bartolome J, Bao J, et al. Hierarchical action and inhibition of plant Dicer-like proteins in antiviral defense. Science, 
2006, 313: 68-71

32 Blevins T, Rajeswaran R, Shivaprasad P V, et al. Four plant Dicers mediate viral small RNA biogenesis and DNA virus induced silencing. Nucleic Acids Res, 2006, 34: 6233-6246

33 Yoshikawa M, Peragine A, Park M Y, et al. A pathway for the biogenesis of trans-acting siRNAs in Arabidopsis. Genes Dev, 2005, 19: 2164-2175

34 Vazquez F, Vaucheret H, Rajagopalan R, et al. Endogenous transacting siRNAs regulate the accumulation of Arabidopsis mRNAs. Mol Cell, 2004, 16: 69-79

35 Peragine A, Yoshikawa M, Wu G, et al. SGS3 and SGS2/SDE1/RDR6 are required for juvenile development and the production of transacting siRNAs in Arabidopsis. Genes Dev, 2004, 18: 2368-2379

36 Baulcombe D. RNA silencing in plants. Nature, 2004, 431: 356-363

37 Harvey J J W, Lewsey M G, Patel K, et al. An antiviral defense role of AGO2 in plants. PLoS ONE, 2011, 6: e14639

38 Takeda A, Iwasaki S, Watanabe T, et al. The mechanism selecting the guide strand from small RNA duplexes is different among Argonaute proteins. Plant Cell Physiol, 2008, 49: 493-500

39 Qu F, Ye X, Morris T J. Arabidopsis DRB4, AGO1, AGO7, and RDR6 participate in a DCL4-initiated antiviral RNA silencing pathway negatively regulated by DCL1. Proc Natl Acad Sci USA, 2008, 105: $14732-14737$

40 Wang X B, Jovel J, Udomporn P, et al. The 21-nucleotide, but not 22-nucleotide, viral secondary small interfering RNAs direct potent antiviral defense by two cooperative Argonautes in Arabidopsis thaliana. Plant Cell, 2011, 23: 1625-1638

41 Montgomery T A, Howell M D, Cuperus J T, et al. Specificity of ARGONAUTE7-miR390 interaction and dual functionality in TAS3 trans-acting siRNA formation. Cell, 2008, 133: 128-141

42 Mi S, Cai T, Hu Y, et al. Sorting of small RNAs into Arabidopsis argonaute complexes is directed by the $5^{\prime}$ terminal nucleotide. Cell, 2008, 133: 116-127

43 Simon-Mateo C, Garcia J A. MicroRNA-guided processing impairs Plum pox virus replication, but the virus readily evolves to escape this silencing mechanism. J Virol, 2006, 80: 2429-2436

$44 \mathrm{Li} \mathrm{H}$, Li W X, Ding S W. Induction and suppression of RNA silencing by an animal virus. Science, 2002, 296: 1319-1321

45 Diaz-Pendon J A, Li F, Li W X, et al. Suppression of antiviral silencing by cucumber mosaic virus $2 \mathrm{~b}$ protein in Arabidopsis is associated with drastically reduced accumulation of three classes of viral small interfering RNAs. Plant Cell 2007, 19: 2053-2063

46 Martin-Hernandez A M, Baulcombe D C. Tobacco rattle virus 16-kilodalton protein encodes a suppressor of RNA silencing that allows transient viral entry in meristems. J Virol, 2008, 82: 4064-4071

47 Du Q S, Duan C G, Zhang Z H, et al. DCL4 targets Cucumber mosaic virus satellite RNA at novel secondary structures. J Virol, 2007, 81: 9142-9151

48 Zhu H, Duan C G, Hou W N, et al. Satellite RNA-derived satsiR-12 targeting the 3'UTR of Cucumber mosaic virus triggers viral RNAs for degradation. J Virol, 2011, 85: 13384-13397

49 Hou W N, Duan C G, Fang R X, et al. Satellite RNA reduces expression of the $2 \mathrm{~b}$ suppressor protein resulting in the attenuation of symptoms caused by Cucumber mosaic virus infection. Mol Plant Pathol, 2011, 12: 595-605
50 Alvarado V, Scholthof H B. Plant responses against invasive nucleic acids: RNA silencing and its suppression by plant viral pathogens. Semin Cell Dev Biol, 2009, 20: 1032-40

51 Díaz-Pendón J A, Ding S W. Direct and indirect roles of viral suppressors of RNA silencing in pathogenesis. Annu Rev Phytopathol, 2008, 46: 303-326

52 Dunoyer P, Voinnet O. The complex interplay between plant viruses and host RNA-silencing pathways. Curr Opin Plant Biol, 2005, 8: 415-423

53 Wang M B. On the role of RNA silencing in the pathogenicity and evolution of viroids and viral satellites. Proc Natl Acad Sci USA, 2004, 101: 3275-3280

54 Devic M, Jaegle M, Baulcombe D. Symptom production on tobacco and tomato is determined by two distinct domains of the satellite RNA of cucumber mosaic virus (strain Y). J Gen Virol, 1989, 70: 2765-2774

55 Masuta C, Takanami Y. Determination of sequence and structural requirements for pathogenicity of a cucumber mosaic virus satellite RNA (Y-satRNA). Plant Cell, 1989, 1: 1165-1173

56 Jaegle M, Devic M, Longstaff M, et al. Cucumber mosaic virus satellite RNA (Y strain): analysis of sequences which affect yellow mosaic symptoms on tobacco. J Gen Virol, 1990, 71: 1905-1912

57 Kuwata S, Masuta C, Takanami Y. Reciprocal phenotype alterations between two satellite RNAs of cucumber mosaic virus. J Gen Virol, 1991, 72: 2385-2389

58 Smith N A, Eamens A L, Wang M B. Viral small interfering RNAs target host genes to mediate disease symptoms in plants. PLoS Pathog, 2011, 7: e1002022

59 Li W X, Ding S W. Viral suppressors of RNA silencing. Curr Opin Biotechnol, 2001, 12: 150-154

60 Moissiard G, Voinnet O. Viral suppression of RNA silencing in plants. Mol Plant Pathol, 2004, 5: 71-82

61 Roth B M, Pruss G J, Vance V B. Plant viral suppressors of RNA silencing. Virus Res, 2004, 102: 97-108

62 Shimura H, Pantaleo V, Ishihara T, et al. A viral satellite RNA induces yellow symptoms on tobacco by targeting a gene involved in chlorophyll biosynthesis using the RNA silencing machinery. PLoS Pathog, 2011, 7: e1002021

63 Manfre A. Importance of coat protein and RNA silencing in satellite RNA/virus interactions. Virology, 2008, 379: 161-167

64 Zhang F, Simon A E. Enhanced viral pathogenesis associated with a virulent mutant virus or a virulent satellite RNA correlates with reduced virion accumulation and abundance of free coat protein. Virology, 2003, 312: 8-13

65 Raja P, Sanville B C, Buchmann R C, et al. Viral genome methylation as an epigenetic defense against geminiviruses. J Virol, 2008, 82: 8997-9007

66 Yang X, Xie Y, Raja P, et al. Suppression of methylation-mediated transcriptional gene silencing by $\beta \mathrm{C} 1-\mathrm{SAHH}$ protein interaction during geminivirus-betasatellite infection. PLoS Pathog, 2011, 7: e1002329

67 Blevins T, Rajeswaran R, Aregger M, et al. Massive production of small RNAs from a non-coding region of Cauliflower mosaic virus in plant defense and viral counter-defense. Nucleic Acids Res, 2011, 39: 5003-5014

Open Access This article is distributed under the terms of the Creative Commons Attribution License which permits any use, distribution, and reproduction in any medium, provided the original author(s) and source are credited. 